\title{
Thermal Indices and Heat stress Assessment in Livestock: Way Forward
}

\author{
V Sejian*1, G Krishnan ${ }^{1}$ and JP Amitha ${ }^{2}$ \\ ${ }^{1}$ ICAR-National Institute of Animal Nutrition and Physiology, India \\ ${ }^{2}$ Academy of Climate Change Education and Research, Kerala Agricultural University, India
}

Received: February 28, 2018; Published: March 09, 2018

*Corresponding author: V Sejian, ICAR-National Institute of Animal Nutrition and Physiology, Hosur Road, Bangalore, India, Email: drsejian@gmail.com

\begin{abstract}
Abbreviations
THI: Temperature Humidity Index; ETI: Equivalent Temperature Index; IRTCI: Integrated Relative Thermal-Comfort Index; HLI: Heat Load Index; AHL: Accumulated Heat Load Index; CCI: Comprehensive Climate Index; RH: Relative Humidity; WS: Wind Speed; Rad: Solar Radiation; Ta: Ambient Temperature
\end{abstract}

\section{Background}

In the changing climate scenario, heat stress is the most detrimental factor which negatively influences livestock production. This may lead to severe economic consequences in livestock industry. The negative impacts of high temperature stress are generally aggravated when it is coupled with high relative humidity. Every animal produce optimally under its own thermoneutral zone. Different factors of climate such as air temperature, humidity, wind velocity, and solar radiation have prominent effect in influencing the homeostasis in animals. The interaction of these climate variables and the animal biological system lead to drastic changes in the production performance of the animal. This has paved the way for the scientific community to look for a range of thermal indices to specific locations to quantify heat stress response in livestock.

\section{Thermal Indices}

Generally the thermal indices are developed keeping in view the high temperature (heat stress) rather than low temperature (cold stress) because of the fact that the internal metabolic heat production in animals are very sensitive to high temperature stress. These bio-climatic indices, apart from diagnosing the stress level in an animal's also reflect their capability to cope with the adversity. The prerequisite for developing such thermal indices are to design them appropriately to suit the local environmental conditions as well the target species in the locality. Emphasis should also be given while developing a thermal index to identify the threshold temperature that negatively affects the animal. Moreover, thermal indices may also act as good indicators of stress related risks and may help to develop appropriate management strategies to improve animal production in such adverse environmental condition.

\section{Temperature-Humidity Index and Associated Draw-} backs

All the thermal indices in livestock are based on relationship between certain climate and production parameters establishing an empirical relationship between them. The majority of the published reports indicate the use of temperature and humidity in the equations that was developed to suit the local environmental and animal needs. Temperature humidity index (THI) is a widely accepted thermal index in livestock and an ideal indicator of heat stress. It mainly depends on the weather parameters like temperature and humidity. Several THI have been designed from a combination of dry bulb, wet bulb and humidity and it provides specific correlation between these parameters and physiological responses. Although, THI index was found to be of relative success in quantifying the stress response in livestock, still there were two major drawbacks associated with such indices. Firstly, THI does not take into account the wind velocity and solar radiation which are considered cardinal weather parameters which hugely influence the stress response in animals. Secondly, the THI does not take into account the accumulated heat load over the period of time when it crosses the threshold values.

Like THI there are few other thermal indices which used some of the primary cardinal weather parameters in the developed 
equation. Equivalent temperature index (ETI) was developed with a goal of determining the impact of heat dissipation on milk production. It included air temperature, humidity and wind speed as the core variables for the cows above their comfort zone. There are several cold and heat stress models for cattle but the drawback for those models were the exclusion of solar radiation as one of the parameter. Another thermal index called integrated relative thermal-comfort index (IRTCI) associates the interactions of the local environment and some animal characteristics. This index establishes that the interaction between climate parameters such as humidity, ambient temperature, radiation and production parameters like fur depth and production level strongly influences the mechanism of thermoregulation.

\section{Advanced Thermal Indices}

There are few indices developed incorporating all four cardinal weather parameters of temperature, relative humidity, solar radiation and wind velocity. Heat load index (HLI), accumulated heat load index (AHL), comprehensive climate index (CCI) are few of these advanced thermal indices which helps to accurately quantify the stress response in livestock. Heat load index (HLI) developed was based on humidity (\%), wind speed (m/s) and black globe temperature $\left({ }^{\circ} \mathrm{C}\right)$ and a base threshold of black globe temperature with $86^{\circ} \mathrm{C}$ was established. Studies establish that the heat is accumulated in cattle during the peak hot hours of a day due to the whole day exposure to sun. This heat dissipates during the night hours when the environment is cooler but insufficient cooling and hotter consecutive day could result in heat accumulation (accumulated heat load) in animals. Apart from HLI, accumulated heat load index (AHL) was developed to understand the extent of risks related with further exposure of animal to a higher temperature. This index is based on the THI hours (where as THI hour model accounts for the intensity and duration of heat stress) and it indicates that the animal is under severe stress when it is subjected to a temperature higher than HLI for a substantial time period. A recently developed comprehensive climate index (CCI) integrates the climate parameters such as relative humidity (RH), wind speed (WS), solar radiation (Rad) and ambient temperature (Ta) estimates the impact of heat stress on cattle. This index is pertinent for a wide range of environments.

\section{Way Forward}

Sufficient refinements are needed in the existing thermal indices to suit the needs of local environment. More indices incorporating all cardinal weather parameters are needed. These indices should be teller made to suit the local environmental conditions. Further, such indices should be species specific taking into account the response mechanisms of various animals. Such developed advanced thermal indices must also be specific to agro-ecological zone and must be developed in such a way to suit to the needs with few correction factors to the similar agro-ecological zone elsewhere.

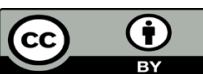

This work is licensed under Creative Commons Attribution 4.0 License

Submission Link: https://biomedres.us/submit-manuscript.php

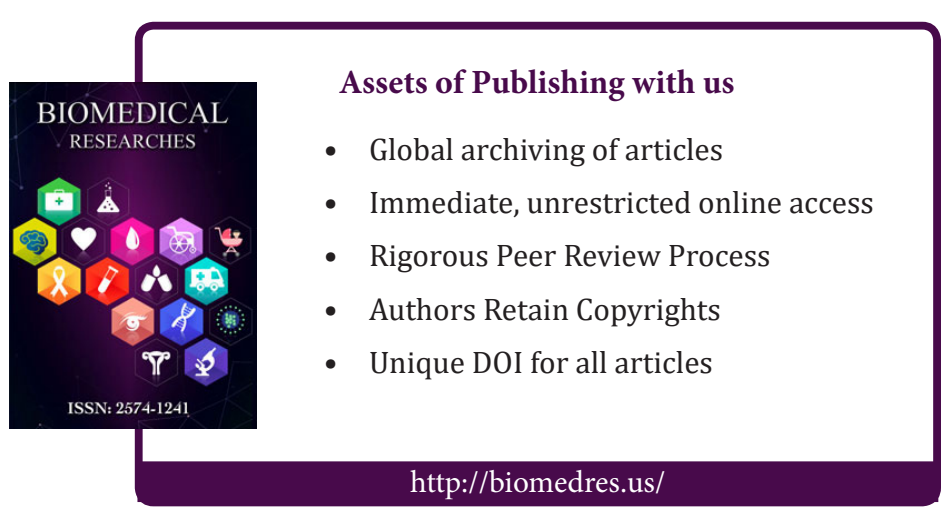

